\title{
Speed evolution of fast CME/shocks with SOHO/LASCO, WIND/WAVES, IPS and in-situ WIND data: analysis of kilometric type-II emissions
}

\author{
A. Gonzalez-Esparza and E. Aguilar-Rodriguez \\ Instituto de Geofisica, Universidad Nacional Autonoma de Mexico, Morelia Mich., Mexico \\ Received: 1 June 2009 - Revised: 1 August 2009 - Accepted: 7 October 2009 - Published: 21 October 2009
}

\begin{abstract}
Fast CME/shocks propagating in the interplanetary medium can generate kilometric Type II (km-TII) radio emissions at the local plasma frequency and/or its harmonic, so these radio emissions provide a means of remotely tracking CME/shocks. We apply a new analysis technique, using the frequency drift of km-TII spectrum obtained by the Thermal Noise Receiver (TNR) of the WIND/WAVES experiment, to infer, at some adequate intervals, the propagation speed of six CME/shocks. We combine these results with previously reported speeds from coronagraph white light and interplanetary scintillation observations, and in-situ measurements, to study the temporal speed evolution of the six events. The speed values obtained by the km-TII analysis are in a reasonable agreement with the speed measurements obtained by other techniques at different heliocentric distance ranges. The combination of all the speed measurements show a gradual deceleration of the CME/shocks as they propagate to $1 \mathrm{AU}$. This new technique can be useful in studying the evolution of fast $\mathrm{CME} /$ shocks when adequate intervals of $\mathrm{km}$-TII emissions are available.
\end{abstract}

Keywords. Interplanetary physics (Interplanetary shocks) Radio science (Radio wave propagation) - Solar physics, astrophysics, and astronomy (Radio emissions)

\section{Introduction}

Coronal Mass Ejections (CMEs) are one of the main manifestations of solar activity. Fast CMEs drive interplanetary shocks and can produce strong geomagnetic storms. The study of these events is very important for space weather purposes and the physical phenomena involved. If we can

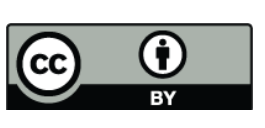

Correspondence to:

A. Gonzalez-Esparza

(americo@geofisica.unam.mx) understand the kinematics of fast CMEs and their shocks in the interplanetary medium, then we could predict the arrival of potential hazardous halo CMEs to the Earth. However, to track these events from near the Sun to $1 \mathrm{AU}$ is a very difficult task with current observational capabilities. To quantify CME kinematics, we need to combine the velocity measurements by different instruments, at different times, covering different heliocentric distances.

We will consider a "fast CME" as a CME with an initial speed well above (supermagnetosonically) the ambient solar wind speed which drives an interplanetary shock. It is well known that fast CME-driven shocks decelerate significantly as they propagate in the interplanetary medium and reach 1 AU (Gosling et al., 1968). Comparing near-Sun plane-ofsky CME speeds by the Large Angle Spectroscopic Coronagraph (LASCO) (Brueckner et al., 1995) with their interplanetary counterparts at $1 \mathrm{AU}$ shows that fast CMEs decelerate as they propagate through interplanetary space (Gopalswamy et al., 2000). For a fast CME, its initial LASCO speed is always faster than its corresponding mean $1 \mathrm{AU}$ transit speed, which is faster than its $1 \mathrm{AU}$ local speed (in-situ measurement).

The interplanetary scintillation technique (IPS) uses the scattering of radio waves from distant sources (e.g., quasars, galaxies, etc.) produced by electron density irregularities in the solar wind by measurement of amplitude (or phase) scintillations. A travelling fast CME/shock generates an increment of the local density turbulence by the compression of the solar wind between the shock and its CME-driver (i.e., the sheath region). IPS allows us to identify this portion of shocked solar wind in front of the fast CME. Studies combining LASCO white light observations, IPS, and insitu measurements show that fast CME/shocks present a twostep deceleration process in the IP medium: an initial phase of small deceleration until they reach a certain heliocentric distance ( $\sim 60-120$ solar radii) from which it increases and the $\mathrm{CME} /$ shock tends to become equal to the ambient wind

Published by Copernicus Publications on behalf of the European Geosciences Union. 
velocity (Manoharan, 2006; Tokumaru et al., 2006; Pohjolainen et al., 2007).

\subsection{Type II radio bursts}

It is also possible to track fast CME/shocks using electromagnetic wave observations by a spacecraft or satellite. Solar radio bursts of Type II are characterized by a narrow band of intense radiation that drifts with time and distance from the Sun. Type II solar radio bursts are produced by the excitation of plasma waves in the ambient medium by a shock wave propagating outward from the Sun. These Type II emissions occur at the fundamental $(\mathrm{F})$ and/or harmonic $(\mathrm{H})$ of the plasma frequency, $f$, which is related to the square root of the electron plasma density, $n$, in the source region: $f=a \sqrt{n}$ (where $a=9$ for the fundamental emission (F), $a=18$ for the harmonic emission $(\mathrm{H})$, and $\left.f[\mathrm{kHz}], n\left[\mathrm{~cm}^{-3}\right]\right)$.

These Type II radio emissions usually start at frequencies below $150 \mathrm{MHz}$, where the disturbance is just a few solar radii away from the Sun and they may extend down to the kilometric domain, slowly drifting to lower frequencies all the way to $1 \mathrm{AU}$, where the local plasma frequency of the solar wind is $\sim 25 \mathrm{kHz}$. Type II radio bursts are classified according to their wavelength regime as: metric $(\mathrm{m})$, decameter/hectometric $(\mathrm{DH})$, and kilometric $(\mathrm{km})$ bands.

It is now well established that DH to km wavelength (interplanetary) Type II radio emissions are caused by the propagation of fast $\mathrm{CME} /$ shocks through the interplanetary medium (Cane et al., 1987). The frequency-drifting Type II radio emissions can, in principle, provide continuous tracking of a CME/shock from the solar corona all the way to $1 \mathrm{AU}$. Therefore, these radio observations can be used to reconstruct the speed profile for a CME-driven shock (Reiner et al., 1998). However, not necessarily all the CME/shocks generate Type II radio emissions (Gopalswamy et al., 2008).

Reiner et al. (1998) showed that the radio emission from a given source region along the shock front is expected to be organised along a straight line, assuming that the speed of the shock is approximately constant. Hence, the slope of this line may be determined as

slope $=\frac{v}{a \sqrt{n_{o}} R_{0}}$,

where $v$ is the shock speed, $n_{o}$ is the density normalised to $1 \mathrm{AU}, R_{0}=1.5 \times 10^{8} \mathrm{~km}, a=9$ if the emission is fundamental (F), and $a=18$ if the emission is harmonic (H).

Since the interplanetary density decreases with the square of the heliocentric distance, the CME/shock will generate radio emissions at decreasing frequencies as it propagates through interplanetary space. The observed frequency drift rate of Type II radio emissions generated by CME-driven shocks, provides a direct measurement of the shock speed (Reiner et al., 1998; Cremades et al., 2007). Hence the shock speed $(v)$ is computed as:

$v=$ (slope) $a R_{0} \sqrt{n_{o}}$.
Equation (2) can be used to infer the shock propagation speed $(v)$ if some assumptions are satisfied. We need to know the value of the solar wind density $\left(n_{o}\right)$ at $1 \mathrm{AU}$. We need a well defined Type II radio emission associated with the propagation of the interplanetary shock at a constant speed through an homogeneous ambient solar wind which density decreases with the square of the heliocentric distance. We need to calculate the slope of the central frequency emission of the Type II event.

The purpose of this paper is to report the results of a study on the propagation of six fast CMEs detected by LASCO, where each was driven an interplanetary shock, to track their temporal speed evolution. We apply a new technique to deduce the propagation speed of these events from analyzing the slope of kilometric Type II (km-TII) burst drifts. We use this low frequency emissions because we are interested in the speed evolution of these disturbances far away from the Sun in a region where the solar wind density decreases with the square of the heliocentric distance. We combine these speed results with previously published plane-of-the-sky LASCO CME, IPS, and shock and ejecta in-situ speed measurements by other instruments to illuminate the temporal evolution of the six events. The study of these fast $\mathrm{CME} /$ shocks provides more information to track when (where) fast CME/shocks decelerate as they propagate through the interplanetary medium.

\section{Data analysis}

\subsection{Event selection criteria}

The radio observations analyzed in this report were acquired by the WAVES experiment on board the WIND spacecraft. The WAVES experiment has several sensitive radio receivers (RAD2, RAD1, and TNR) that cover the frequency range from $4 \mathrm{kHz}$ to $\sim 14 \mathrm{MHz}$ (Bougeret et al., 1995). We analyze 1-min resolution data from the Thermal Noise Receiver (TNR), which covers a frequency range from 4 to $245 \mathrm{kHz}$ in five logarithmically-spaced frequency bands. Each of these bands is divided into either 32 or 16 logarithmically-spaced channels.

Reiner et al. (2007) investigated the kinematics of 42 $\mathrm{CME} /$ shocks associated with solar Type-II radio bursts detected by WIND/WAVES during solar cycle 23 . The interplanetary counterparts of all these CMEs were detected in-situ at $\mathrm{L}_{1}$ by WIND and some of them caused geomagnetic storms as reported elsewhere (Richardson and Cane, 1995; Manoharan et al., 2004; Reiner et al., 2007). We used Reiner et al. (2007) event list to investigate the corresponding WAVES/TNR dynamic spectra and to determine the feasibility of applying the technique that we describe in the next sub-section. After inspecting the TNR data, we reduced the initial list to six fast CMEs (Table 1). This is a period of very high transient solar activity and for the six CMEs in the 
Table 1. (1) CME date and (2) time detected by LASCO, (3) plane-of-sky LASCO velocity linear fit, (4) velocity from interplanetary scintillation (IPS) measurements, (5) shock date and (6) time detected by WIND, (7) shock local velocity, (8) shock transit velocity to 1 AU, (9) ICME date and (10) time detected by WIND, (11) average ICME local velocity, (12) ICME transit velocity to 1 AU.

\begin{tabular}{|c|c|c|c|c|c|c|c|c|c|c|c|}
\hline 1 & 2 & 3 & 4 & 5 & 6 & 7 & 8 & 9 & 10 & 11 & 12 \\
\hline $\begin{array}{c}\text { CME } \\
\text { date }\end{array}$ & $\begin{array}{l}\mathrm{CME} \\
\text { time }\end{array}$ & $\begin{array}{r}V_{\mathrm{cme}}^{\mathrm{a}} \\
{[\mathrm{km} / \mathrm{s}]}\end{array}$ & $\begin{array}{c}V_{\mathrm{ips}}^{\mathrm{b}, \mathrm{c}} \\
{[\mathrm{km} / \mathrm{s}]}\end{array}$ & $\begin{array}{l}\text { shock } \\
\text { date }\end{array}$ & $\begin{array}{c}\text { shock } \\
\text { time }\end{array}$ & $\begin{array}{l}V_{\text {shock }}^{\mathrm{d}, \mathrm{e}} \\
{[\mathrm{km} / \mathrm{s}]}\end{array}$ & $\begin{array}{l}\overline{V_{\text {shock }}} \\
{[\mathrm{km} / \mathrm{s}]}\end{array}$ & $\begin{array}{c}\text { ICME } \\
\text { date }\end{array}$ & $\begin{array}{l}\text { ICME } \\
\text { time }\end{array}$ & $\begin{array}{c}V_{\mathrm{icme}}^{\mathrm{f}} \\
{[\mathrm{km} / \mathrm{s}]}\end{array}$ & $\begin{array}{r}\overline{V_{\text {icme }}} \\
{[\mathrm{km} / \mathrm{s}]}\end{array}$ \\
\hline 6 Jun 2000 & $15: 54$ & 1119 & $935^{\mathrm{c}}$ & 8 Jun 2000 & 08:41 & $860^{\mathrm{d}}$ & 1015 & 8 Jun 2000 & $12: 00$ & 610 & 938 \\
\hline $14 \mathrm{Jul} 2000$ & $10: 54$ & 1674 & $1070^{\mathrm{c}}$ & $15 \mathrm{Jul} 2000$ & $14: 15$ & $861^{\mathrm{d}}$ & 1513 & $15 \mathrm{Jul} 2000$ & $19: 00$ & 740 & 1289 \\
\hline 29 Mar 2001 & $10: 26$ & 942 & $782^{b}$ & 31 Mar 2001 & $22: 56$ & $617^{\mathrm{e}}$ & 684 & 1 Apr 2001 & 04:00 & 600 & 631 \\
\hline 26 Apr 2001 & $12: 30$ & 1006 & $470^{\mathrm{b}}$ & 28 Apr 2001 & $04: 31$ & $820^{\mathrm{e}}$ & 1034 & 28 Apr 2001 & 14:00 & 540 & 836 \\
\hline 4 Nov 2001 & $16: 35$ & 1810 & $1267^{\mathrm{c}}$ & 6 Nov 2001 & $01: 25$ & $742^{\mathrm{e}}$ & 1260 & 6 Nov 2001 & 21:00 & 600 & 790 \\
\hline $25 \mathrm{Jul} 2004$ & $14: 54$ & 1333 & N/D & $26-07-2004$ & $22: 30$ & $1100^{\mathrm{d}}$ & 1310 & $27 \mathrm{Jul} 2004$ & 02:00 & 870 & 1179 \\
\hline
\end{tabular}

${ }^{a}$ SOHO-LASCO CME catalog (http://cdaw.gsfc.nasa.gov/CME_list/index.html); ${ }^{b}$ Manoharan et al. (2006); ${ }^{c}$ Tokumaru et al. (2006); ${ }^{d}$ Reiner et al. (2007); ${ }^{\mathrm{e}}$ Manoharan et al. (2004); ${ }^{\mathrm{f}}$ List of Richarson/Cane ICMEs in 1996-2007 (http://www.ssg.sr.unh.edu/mag/ace/ACElists/ ICMEtable.html)

study, there were several slower ones preceding their launch (Manoharan et al., 2004). This implies that for all these CMEs the ambient solar wind was highly inhomogeneous, affecting the propagation of the CME/shocks. The characteristics of the Type II bursts were obtained from the online list from WIND/WAVES (http://lep694.gsfc.nasa.gov/waves/ waves), maintained by M. L. Kaiser.

All the CMEs in Table 1 were reported as halo. Columns 1 and 2 show the CME date and time as they appear in the SOHO-LASCO CME catalog. Column 3 shows the initial CME speed $\left(V_{\text {cme }}\right)$ estimated by fitting a straight line to the height-time measurements in the LASCO field of view (Yashiro et al., 2004). Column 4 shows the speed of the interplanetary counterpart $\left(V_{\mathrm{ips}}\right)$ as reported from IPS observations. These $V_{\mathrm{ips}}$ values were obtained whether by the Solar-Terrestrial Laboratory (STELab) as reported by Tokumaru et al. (2006) or by the Ooty Radio Telescope (ORT) as reported by Manoharan (2006). Since the two observatories have different antenna systems, they estimated the $V_{\mathrm{ips}}$ using different methods. The interplanetary counterparts of the six CMEs were detected in-situ by WIND. Columns 5 and 6 present the shock-wave date and time as detected by the WIND spacecraft at $\mathrm{L}_{1}$ (Reiner et al., 2007). Column 7 shows the local shock speed $\left(V_{\text {shock }}\right)$ inferred from in-situ WIND data by employing the Rankine-Hugoniot relations as reported by Reiner et al. (2007) and/or Manoharan et al. (2004). Column 8 is the mean shock transit speed $\left(\overline{V_{\text {shock }}}\right)$ from taking the LASCO-CME observations as the initial time and the shock WIND observations as the final time. Columns 9 and 10 show the ICME date and time as reported by (Richardson and Cane, 1995). Column 11 shows the local mean ICME speed $\left(V_{\text {icme }}\right)$, from averaging the bulk speed along the whole ejecta in the spacecraft data Richard- son and Cane (1995). Finally, column 12 shows the ICME transit speed $\left(\overline{V_{\text {icme }}}\right)$, from taking the LASCO-CME observations as the initial time and the WIND-ICME observations as the final time. Note that in the six CMEs, the local shock speed $\left(V_{\text {shock }}\right)$ is higher than the ICME local average speed $\left(\mathrm{V}_{\text {icme }}\right)$. However, comparing the mean transit speeds of the shock $\left(\overline{V_{\text {shock }}}\right)$ and the ICME $\left(\overline{V_{\text {icme }}}\right)$ with their corresponding local speeds, we notice a clear deceleration of both disturbances: the transit speeds are faster than the local ones.

\subsection{Methodology}

We present a case study to explain the technique in detail. Figure 1 shows the $\mathrm{km}$-TII analysis of the first CME/shock in Table 1. On 6 June 2000, WIND/WAVES detected a Type II radio burst starting at $\sim 15: 20 \mathrm{UT}$ at $\sim 14 \mathrm{MHz}$ and drifting down to $\sim 40 \mathrm{kHz}$ at $\sim 09: 00 \mathrm{UT}$ on 8 June. According to the WIND/WAVES Type II list, the event is reported as a fundamental/harmonic pair at the start, then sometimes very intense single tone. Figure 1a shows the corresponding kmTII emission detected by the WAVES/TNR receiver. The $\mathrm{km}$-TII burst feature is observed at $\sim 13: 50$ UT on 7 June at $\sim 100 \mathrm{kHz}$, drifting slowly down to $\sim 60 \mathrm{kHz}$ at $\sim 00: 30 \mathrm{UT}$ on 8 June. The $\mathrm{km}$-TII burst is characterized by a single tone with intervals of very intense emission, which last several hours.

In general, one of the problems in analyzing Type II solar burst data is to identify the fundamental emission lane in the dynamic radio spectrum. We apply the technique by Aguilar-Rodriguez et al. (2005) to infer this fundamental frequency for every 1-min TNR spectrum. We began the analysis by isolating the Type-II event from the TNR dynamic spectrum by setting to zero any emission outside the TypeII burst feature. Figure 1b shows the Type-II burst isolated 


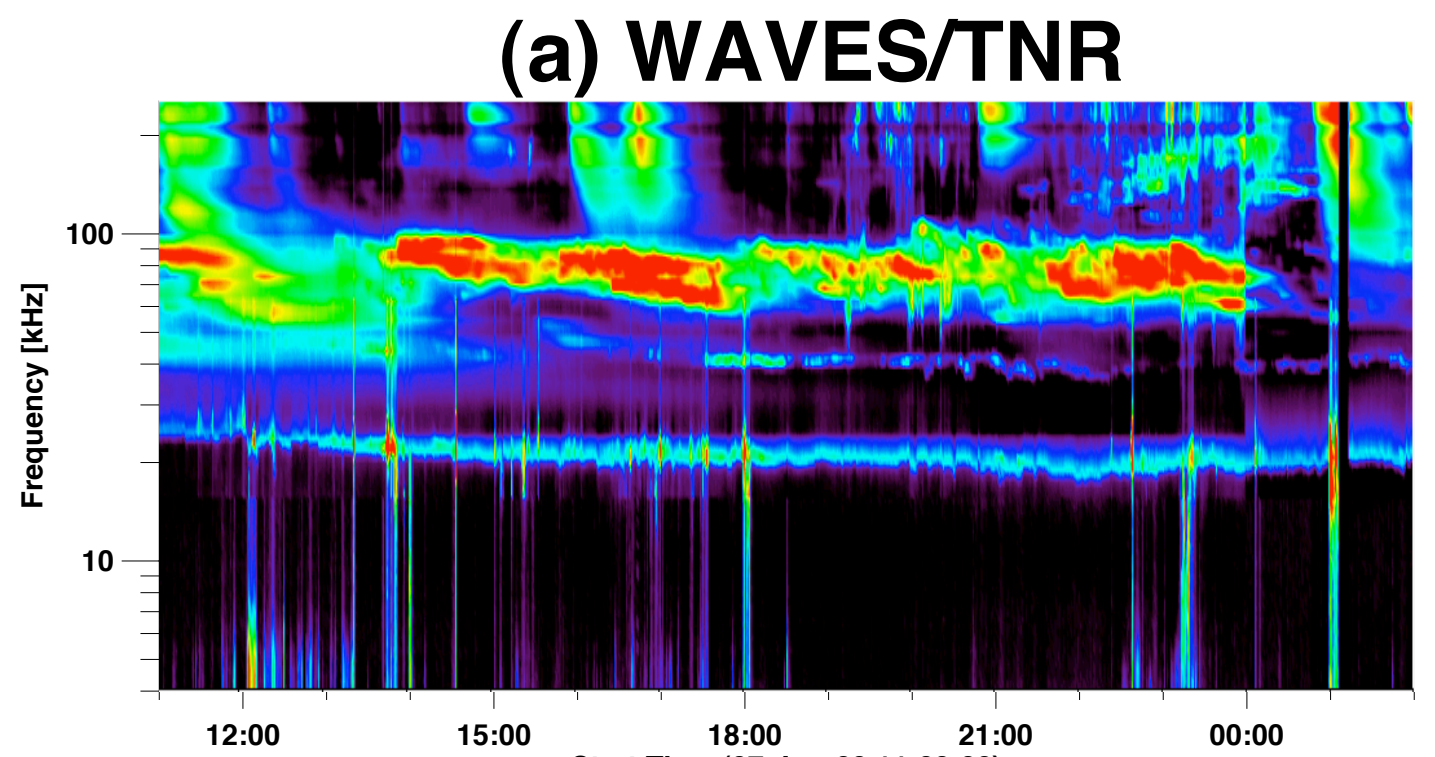

(b)
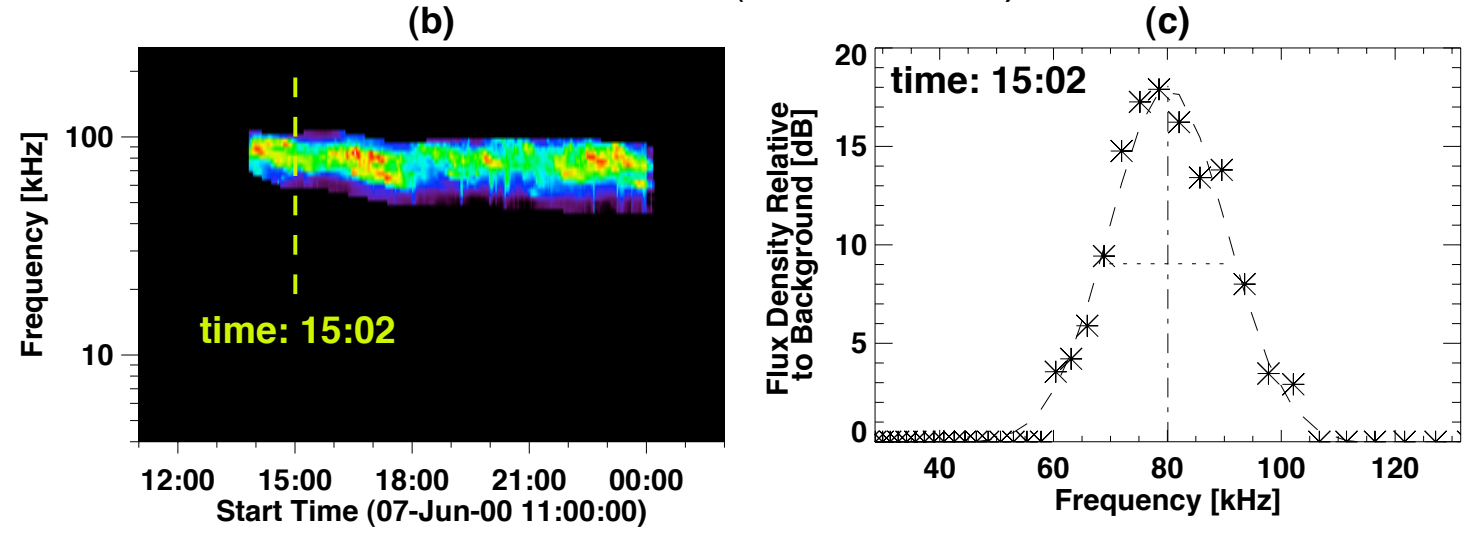

(d)
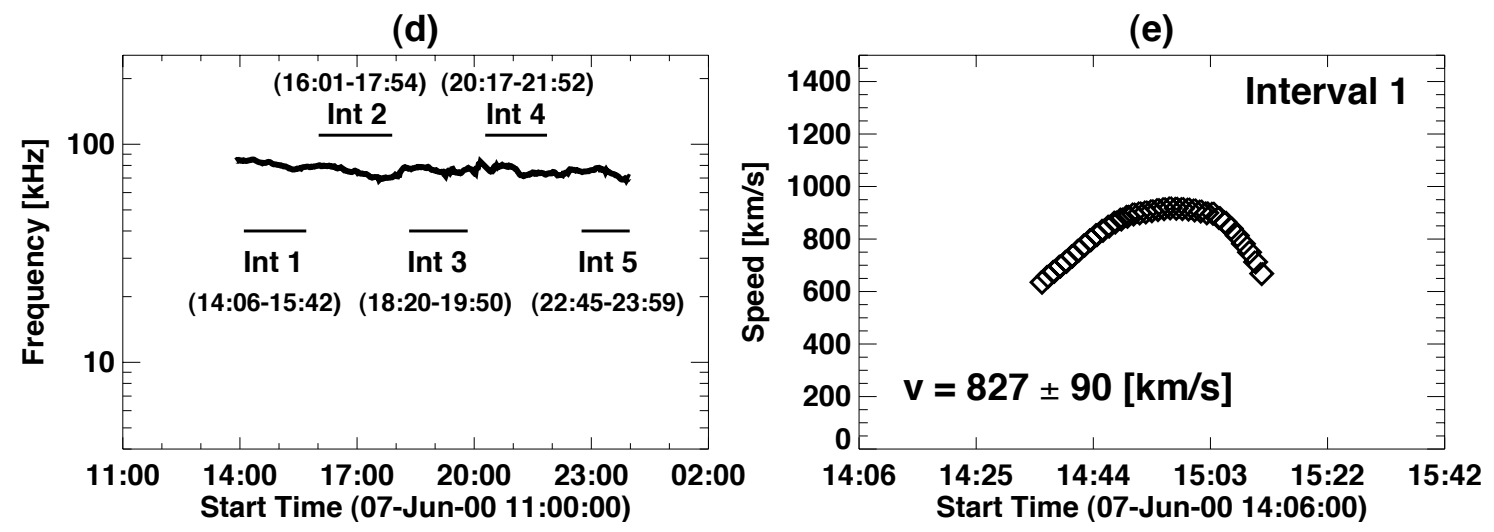

Fig. 1. Case study, central frequency drift and speed deduction. LASCO/CME on 6 June 2000. (a) WAVES/TNR dynamic spectrum associated with the propagation of the CME/shock. (b) Isolated Type II solar burst, (dotted line) temporal cut to perform the central frequency analysis. (c) Flux density versus frequency for 1-min TNR spectrum at 15:02 UT. The gaussian fit gives the central frequency. (d) Central frequency versus time. The horizontal lines show the five intervals to perform the km-TII slope analysis. (e) Shock speed results appliying the $\mathrm{km}$-TII slope analysis in all the sub-intervals within interval 1 . The shock speed and its uncertainty are given by the mean value and the standard deviation of the distribution of speed values (see text). 
from the dynamic spectrum and a vertical line indicating a time cut (15:02 UT). Then we analyzed every TNR spectrum data to obtain its spectral properties. Figure 1c shows the flux density versus the frequency of the Type-II radio burst at 15:02 UT (asterisks) and a Gaussian fit to the flux-density distribution (dashed line). This Gaussian approximation to the flux density distribution gives us, when there is a good fit, the central frequency (dash-dotted line) which is the fundamental emission lane in the radio spectrum at the given time. This Gaussian fit to the flux-density distribution at every time cut of the spectrum gives us a criteria to decide whether the time cut in the spectrum has a well-defined central emission or not. There are some spectra with two or three maxima in the flux density distribution, in this case we discard the spectrum and do not consider any central emission for this time. This procedure cleans the data from emission contamination. Only data from those cuts in time with good Gaussian fits were chosen.

Once the central frequency emissions were obtained, the frequency drift associated with the km-TII radio emission were studied. Figure 1d shows the central emission frequency versus time for the CME on 6 June 2000. The time interval is about eight hours. Although the frequency shows a tendency to decrease with time, the drift is not smooth and there are regions where the frequency increases. In these regions, the methodology described above to infer the $\mathrm{CME} /$ shock speed using the $\mathrm{km}$-TII frequency drift cannot be applied. These local increments in frequency are related to spectral contamination by Type III events, AKR or inhomogeneities in the ambient plasma density. Therefore, event by event, we needed to eye-scan the temporal evolution of the central emission frequency, to find those intervals where there is a clean km-TII frequency drift which is likely associated with the velocity of the CME/shock. We need to satisfy as possible the assumptions to apply Eq. (2). Figure 1d shows the five "adequate" intervals that we selected for this event to perform the km-TII speed analysis. The selected intervals have a duration longer than one hour.

The next step in the analysis is to infer the slope of the frequency drift in the selected intervals and using Eq. (2) (choosing a value for $n_{o}$ and if it is the $\mathrm{F}$ or $\mathrm{H}$ emission) to get the shock speed. The frequency slope was obtained by applying a linear fit to the central frequency points in the interval. In order to test the stability of the solutions, we computed the frequency slope of short sub-intervals of a few minutes (530) along the interval. We applied Eq. (2) to every slope and obtained a very large dispersion of shock speeds. However, upon increasing the duration of the sub-intervals, the speed solutions tended to converge to certain values. This indicates that the sub-intervals must be longer than 60 (90-120) min. Knowing this problem with the stability of the shock speed solutions, we require to estimate an associated error. Then we calculated the slope for every set of $60(90-120)$ consecutive central frequency points in the interval. This gives an ensemble of speed solutions associated with all the sub-
Table 2. Time intervals for the km-TII shock speed analysis. Initial and final interval time, length of the sub-intervals $(\Delta t)$ for the speed analysis, and shock speed result. For all the events we assumed $n_{o}=5$ in Eq. (2) as noted in the text.

\begin{tabular}{ccrr}
\hline initial time & final time & $\begin{array}{c}\Delta t \\
{[\mathrm{~min}]}\end{array}$ & \multicolumn{1}{c}{$\begin{array}{c}\text { velocity } \\
{[\mathrm{km} / \mathrm{s}]}\end{array}$} \\
\hline \multicolumn{5}{c}{ LASCO-CME 6 Jun 2000 } \\
7 Jul 2000 14:06 & 7 Jul 2000 15:42 & 60 & $827 \pm 90$ \\
7 Jul 2000 16:01 & 7 Jul 2000 17:54 & 60 & $1062 \pm 81$ \\
7 Jul 2000 18:20 & 7 Jul 2000 19:50 & 60 & $760 \pm 40$ \\
7 Jul 2000 20:17 & 7 Jul 2000 21:52 & 60 & $1091 \pm 167$ \\
7 Jul 2000 22:45 & 7 Jul 2000 23:59 & 60 & $1119 \pm 288$ \\
LASCO-CME 14 Jul 2000 & & \\
14 Jul 2000 20:11 & 14 Jul 2000 21:22 & 60 & $901 \pm 10^{\mathrm{a}}$ \\
15 Jul 2000 01:30 & 15 Jul 2000 03:42 & 120 & $1396 \pm 30^{\mathrm{a}}$ \\
15 Jul 2000 01:54 & 15 Jul 2000 04:12 & 120 & $1384 \pm 24^{\mathrm{a}}$ \\
LASCO-CME 29 Mar 2001 & & \\
29 Mar 2001 18:21 & 29 Mar 2001 19:28 & 60 & $1274 \pm 20$ \\
LASCO-CME 26 Apr 2001 & & \\
27 Apr 2001 03:31 & 27 Apr 2001 05:42 & 120 & $926 \pm 10$ \\
27 Apr 2001 14:57 & 27 Apr 2001 17:24 & 120 & $909 \pm 50$ \\
27 Apr 2001 19:00 & 27 Apr 2001 21:30 & 120 & $544 \pm 10$ \\
LASCO-CME 4 Nov 2001 & & \\
5 Nov 2001 20:08 & 5 Nov 2001 22:27 & 120 & $934 \pm 20^{\mathrm{a}}$ \\
6 Nov 2001 00:18 & 6 Nov 2001 01:39 & 60 & $1090 \pm 50^{\mathrm{a}}$ \\
6 Nov 2001 02:26 & 6 Nov 2001 03:47 & 60 & $658 \pm 24^{\mathrm{a}}$ \\
LASCO-CME 25 Jul 2004 & & \\
26 Jul 2004 04:18 & 26 Jul 2004 07:56 & 90 & $1325 \pm 72$ \\
26 Jul 2004 11:54 & 26 Jul 2004 14:15 & 90 & $1864 \pm 94$ \\
\hline
\end{tabular}

a assuming the harmonic frequency component $(\mathrm{H})$ in the $\mathrm{km}$-TII drift (Eq. 2).

intervals. Figure 1e shows the speed solutions for interval 1. The distribution of points shows that, in this case, the speed solutions tend to converge towards the maximum value. We define the $\mathrm{km}$-TII shock speed $(827 \mathrm{~km} / \mathrm{s})$ as the mean of the distribution, and its associated uncertainty $(90 \mathrm{~km} / \mathrm{s})$ as the standard deviation of the distribution.

Table 2 shows the "adequate" intervals that we found for the six events and the km-TII shock speed solutions for every interval. The first two columns present the initial and final time for the intervals, the third column shows the length of the sub-intervals $(\Delta t)$, and the fourth column the inferred speed and its uncertainty as obtained from the km-TII analysis described earlier. We choose for all the events a value of $n_{o}=5 \mathrm{~cm}^{-3}$, which is a good mean value based on the WIND in-situ measurements in the upstream regions of their associated interplanetary shocks at $\mathrm{L}_{1}$. Table 2 indicates if the $\mathrm{F}$ or $\mathrm{H}$ emission was selected for every event. The intervals have a duration between 1.6 and $3.6 \mathrm{~h}$.

The technique that we applied to analyze the km-TII emissions can be summarized as follows: 1) we looked for intervals where the $\mathrm{km}$-TII emission was not contaminated by 


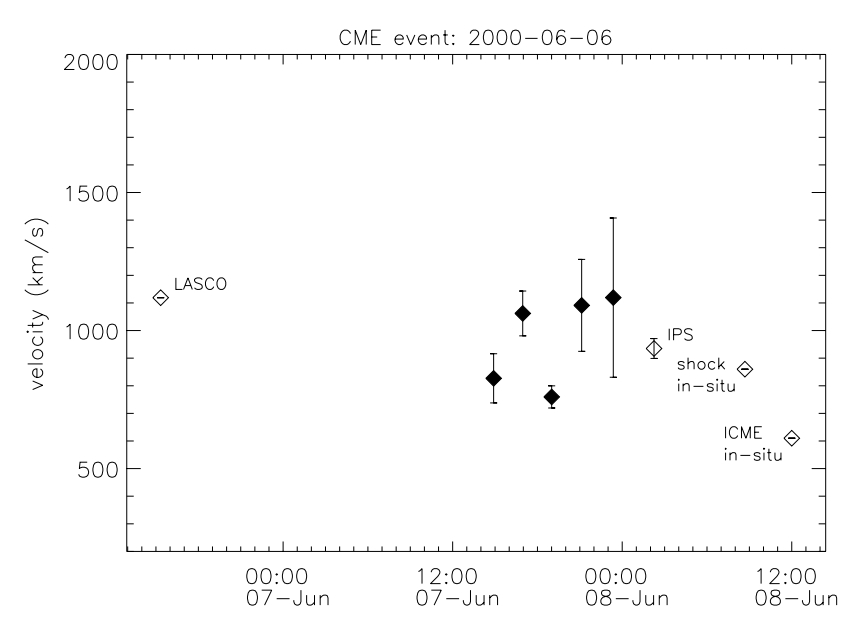

Fig. 2. Speed temporal evolution of the 6 June 2000 LASCO CME. The shock speeds obtained from the analysis of the radio kilometric Type II burst drifts (solid cases) are compared with whitelight (LASCO), interplanetary scintillation (IPS), and in-situ (from WIND plasma and magnetic field data) measurements (see text).

another kind of emission (i.e., Type III radio bursts, auroral kilometric radiation (AKR), etc.); 2) we isolated the km-TII emission; 3) we applied a Gaussian fit , at every spectral data, to the profile of the flux density versus frequency to obtain its spectral properties (central frequency, width and intensity); 4) we only considered those spectra with a well-defined central frequency; 5) we obtain the slope of the central frequency drift by a applying a linear fit at some arbitrary interval; 6) we use Eq. (2) and the slope of the central frequency emission to estimate the shock speed; 7) we apply 5) and 6) at different contiguous intervals to obtain a shock speed distribution; 8) we report the mean value of the shock speeds and its standard deviation as the associated error.

\section{Results}

The temporal speed evolution of the six CME/shocks is reported in Table 1.

\section{June 2000 LASCO CME}

Figure 2 shows the speed evolution of the CME on 6 June 2000. We use this event as the case study in Fig. 1 to show the km-TII speed analysis. The initial LASCO-CME speed was practically constant. The SOHO/LASCO CME catalog reports that the second order fit of the height-time plot gives a very small acceleration of $1.5 \mathrm{~m} / \mathrm{s}^{2}$. The WIND/WAVES list reports a frequency range of $\sim 14 \mathrm{MHz}$ to $40 \mathrm{kHz}$ for the Type-II spectrum. We selected 5 time intervals to apply the $\mathrm{km}$-TII speed analysis (Table 2), and assumed fundamental emission (F) in the frequency drift (Eq. 2). The five shock speeds from the km-TII analysis show reasonable values for

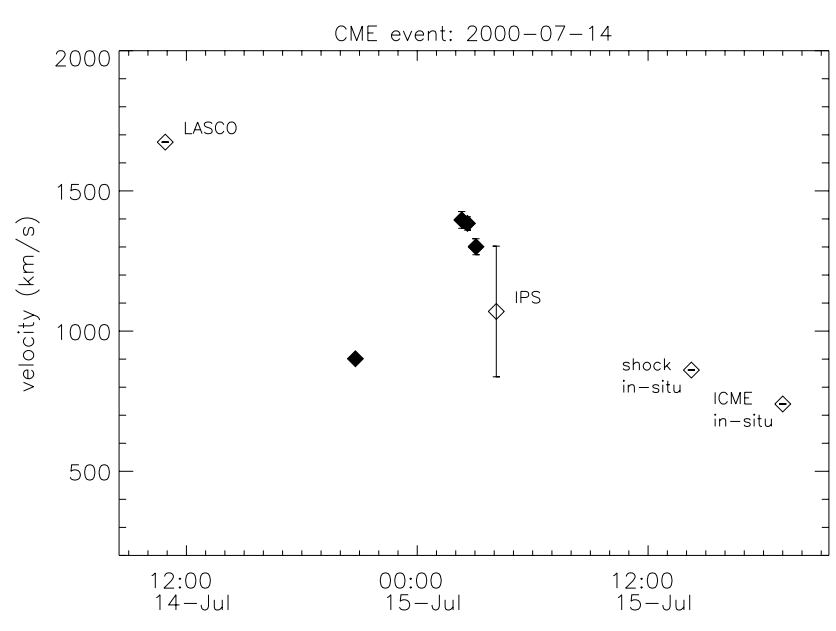

Fig. 3. Speed temporal evolution of the 14 July 2000 LASCO-CME. Same format as previous figure.

the evolution of the CME/shock: they have lower values than the initial LASCO-CME speed, and are faster than the mean WIND-local-ICME speed $\left(V_{\text {icme }}=610 \mathrm{~km} / \mathrm{s}\right)$. The combination of the different speed measurements in Fig. 2, in particular, the three $\mathrm{km}$-TII speeds above the STELab-IPS value $(935 \pm 36 \mathrm{~km} / \mathrm{s})$, shows a gradual tendency for the deceleration of the $\mathrm{CME} /$ shock as it propagates to $1 \mathrm{AU}$.

\section{July 2000 LASCO CME}

Figure 3 shows the speed evolution of the very famous Bastille day CME. This event was studied by Manoharan et al. (2001) claiming that this CME/shock presents a twolevel deceleration: (1) a low decline in speed at distances within or about 100 solar radii, and (2) a rapid decrease at larger distances from the Sun. The CME catalog reports a deceleration of $96 \mathrm{~m} / \mathrm{s}^{2}$. This is the highest initial deceleration in the data set. The WIND/WAVES list reports a frequency range of $14 \mathrm{MHz}$ to $80 \mathrm{kHz}$ for the Type II spectrum. We selected 3 intervals to apply the km-TII speed analysis (Table 2). In this case, we assumed harmonic emission (H) in the frequency drift (Eq. 2). The three shock speeds from the km-TII analysis are within the range of the other speed data points. However, based on the speed temporal evolution in Fig. 3, the first speed value of the km-TII analysis is slower than expected. The STELab-IPS velocity $(1070 \pm 233 \mathrm{~km} / \mathrm{s})$ has an intermediate value between the initial LASCO-CME speed $(1674 \mathrm{~km} / \mathrm{s})$ and the WIND-shocklocal speed $\left(V_{\text {shock }}=861 \mathrm{~km} / \mathrm{s}\right)$.

\section{March 2001 LASCO CME}

Figure 4 shows the speed evolution of the LASCO-CME on 29 March 2001. This is the slowest CME in the data set. The CME catalog reports a small acceleration of $3.5 \mathrm{~m} / \mathrm{s}^{2}$. The 


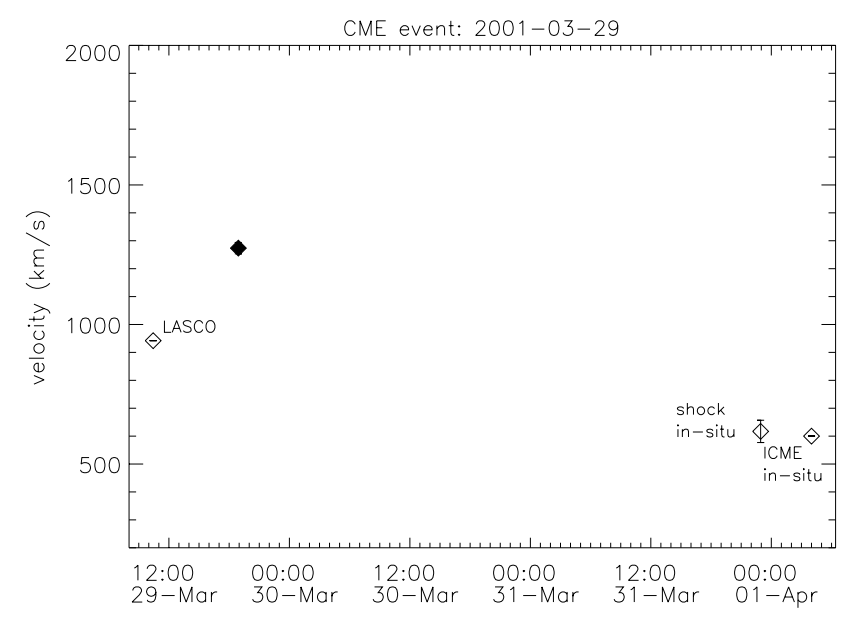

Fig. 4. Speed temporal evolution of the 29 March 2001 LASCOCME. Same format as in Fig. 2.

WIND/WAVES list reports a weak emission and a frequency range of $4 \mathrm{MHz}$ to $60 \mathrm{kHz}$ for the Type II spectrum. We selected only one interval to perform the $\mathrm{km}$-TII speed analysis, and assumed fundamental emission (F) in Eq. (2) (Table 2). Contrary to our expectations, the shock speed from the $\mathrm{km}$-TII analysis $(1274 \pm 20 \mathrm{~km} / \mathrm{s})$ resulted much faster than the initial LASCO-CME speed $(942 \mathrm{~km} / \mathrm{s})$. This result could be bad guessing for $n_{o}$ in Eq. (2) and/or a contamination in the km-TII spectra that we analyzed. The ORTIPS velocity $(782 \mathrm{~km} / \mathrm{s})$ (Manoharan, 2006) does not appear in Fig. 4 since we do not have the time associated with the measurement, but it has an intermediate value between the initial LASCO-CME speed and the $\mathrm{L}_{1}$ WIND-shock-local speed $(617 \mathrm{~km} / \mathrm{s})$.

\section{April 2001 LASCO CME}

Figure 5 shows the speed evolution of the LASCO-CME on 26 April 2001. The CME catalog reports an acceleration of $21.1 \mathrm{~m} / \mathrm{s}^{2}$. This is the highest initial acceleration in the event list. The WIND/WAVES list reports a frequency range of $5 \mathrm{MHz}$ to $20 \mathrm{kHz}$ for the Type II spectrum. There is a gap of several hours in the WIND/TNR data. We selected three intervals to perform the km-TII speed analysis and assumed fundamental emission (F) in Eq. (2) (Table 2). The three $\mathrm{km}$-TII speeds are within the range of the other speed points in the figure. The ORT-IPS speed $(470 \mathrm{~km} / \mathrm{s})$ (Manoharan, 2006) does not appear in Fig. 5 because we do not have the time associated with the measurement. This ORT-IPS speed and the third point of shock speed from the km-TII analysis $(544 \pm 10 \mathrm{~km} / \mathrm{s})$ have a significantly lower value than the reported WIND-shock-local speed $(820 \mathrm{~km} / \mathrm{s})$. It is possible that these two slow speeds might be associated with signatures of different regions than the nose of the CME/shock.

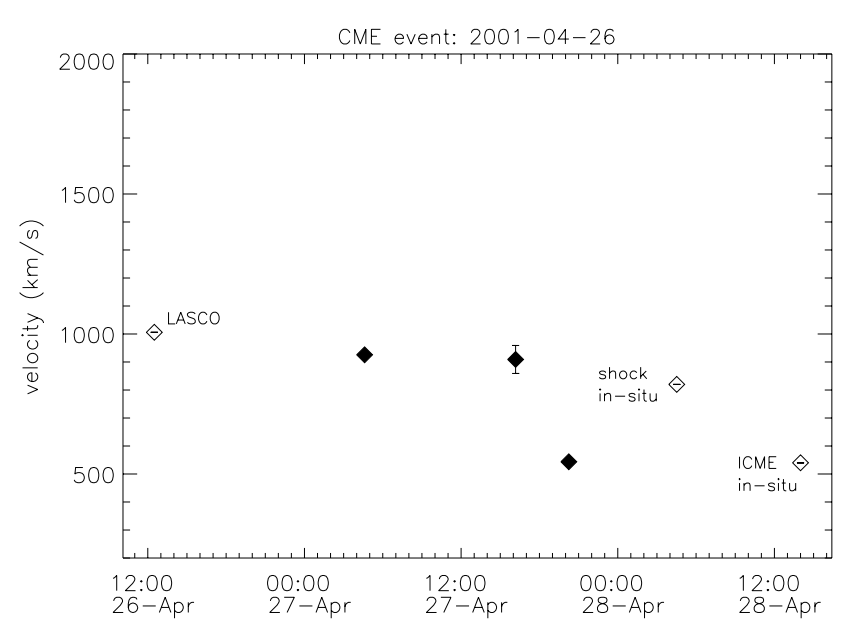

Fig. 5. Speed temporal evolution of the 26 April 2001 LASCOCME. Same format as in Fig. 2.

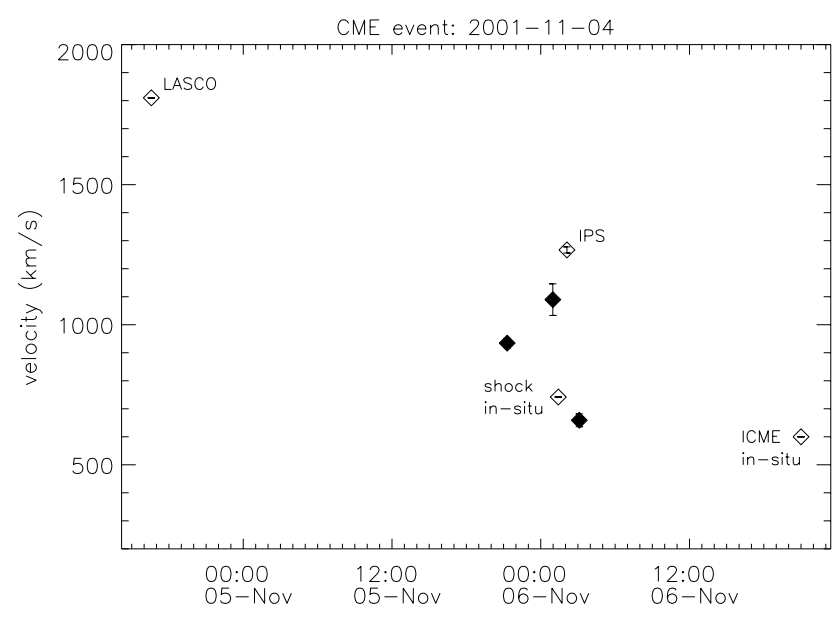

Fig. 6. Speed temporal evolution of the 11 November 2001 LASCO-CME. Same format as in Fig. 2.

\section{November 2001 LASCO CME}

Figure 6 shows the speed evolution of the LASCO-CME on 4 November 2001. This is the fastest CME in the data set. The CME catalog reports a very strong deceleration of $63.4 \mathrm{~m} / \mathrm{s}^{2}$. The WIND/WAVES list reports a frequency range of $14 \mathrm{MHz}$ to $70 \mathrm{kHz}$ for the Type II spectrum. We selected three intervals to perform the km-TII speed analysis and assumed harmonic emission $(\mathrm{H})$ in Eq. (2) (Table 2). The shock speed points of the $\mathrm{km}$-TII analysis are located between the STELab-IPS $(1267 \pm 11 \mathrm{~km} / \mathrm{s})$ and the WINDshock-local $(742 \mathrm{~km} / \mathrm{s})$ speeds. Note that the extended temporal separation between the passing at WIND of the shock and the ICME (Table 1), is about $19.5 \mathrm{~h}$. This is the fastest halo CME in the list so we would expect a faster shock at 


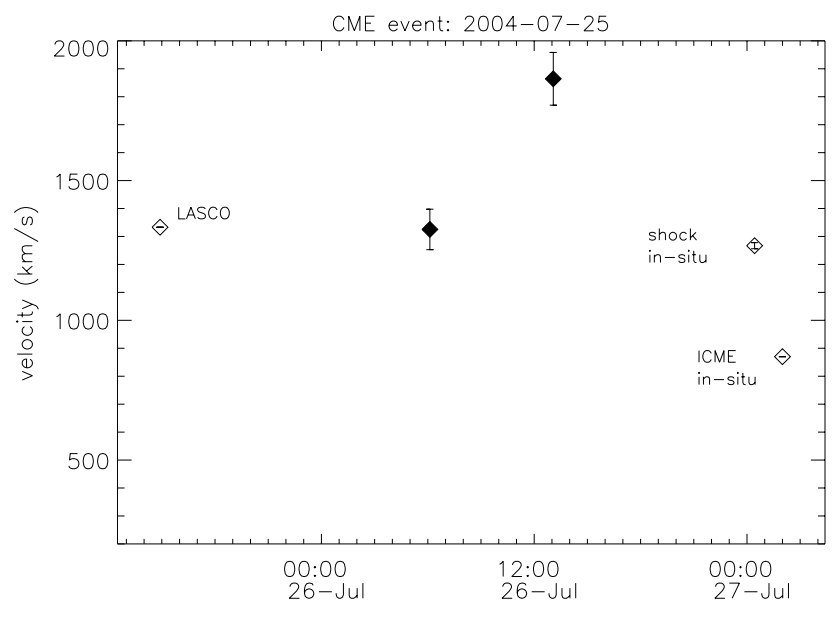

Fig. 7. Speed temporal evolution of the 25 July 2004 LASCO-CME. Same format as in Fig. 2.

$1 \mathrm{AU}$, and also that the shock and the ICME were closer to each other. A possible explanation for this shock-ICME separation is that the nose of the event passed far away from the Earth.

\section{July 2004 LASCO CME}

Figure 7 shows the speed evolution of the LASCO-CME on 25 July 2004. The CME catalog gives an acceleration of $7.0 \mathrm{~m} / \mathrm{s}^{2}$. The WIND/WAVES list reports a frequency range of 1000 to $28 \mathrm{kHz}$ for the Type II spectrum. We selected two intervals to perform the km-TII speed analysis and assumed fundamental emission (F) in Eq. (2) (Table 2). Contrary to our expectations, the second km-TII speed point $(1864 \pm 94 \mathrm{~km} / \mathrm{s})$ is much faster than the reported LASCOCME speed $(1333 \mathrm{~km} / \mathrm{s})$. This result could be a contamination in the km-TII spectra that we analyzed or a bad guessing for $n_{o}$ in Eq. (2). IPS speed data has not been published for this event.

\section{Discussion}

In the study of the six events, we found that in general the shock speeds obtained from the km-TII analysis are within the range of other speed measurements by different instruments. However, in some cases, the km-TII shock speed values inferred from adjacent intervals presents very different values. The reason for that variation might be some contamination in the spectral data in one of the intervals, or the presence of electron density inhomogeneities in the ambient wind. This means that the assumptions required to apply Eq. (2) are not fully satisfied.

It is not straightforward to analyse Type II radio emissions to infer the propagation of fast CME/shocks. To estimate the speed of shock waves in the solar corona we require a static coronal density model, but different electron density models obtain ambiguous results (see Pohjolainen et al., 2007, and references therein). At low frequency emissions (km-TII), we do not have this problem and we can assume that the electron density decays with the square of the heliocentric distance. However not necessarily all the CME/shocks generate $\mathrm{km}$-TII emissions, and for those events which do, the emission is not continuous throughout the whole trajectory and/or presents contamination (e.g., Type III bursts, AKR) in several intervals. We presented a new analysis technique for quantitatively reconstructing the temporal evolution of a fast $\mathrm{CME} /$ shock as it propagates from the Sun out to $1 \mathrm{AU}$ based on the analysis of $\mathrm{km}$-TII radio emissions. The applicability of this technique will depend on the availability and quality of the km-TII spectral data associated with the CME/shock and the validity of the assumptions and constants to apply Eq. (2). In general we find that it is not simple to apply the technique in most of the events.

The events in Table 1 occurred during a period of high solar activity and, in every case, there were between one and four slower preceding CMEs propagating outward within a time window of a few hours. The ambient wind was structured with fluctuations in solar wind density. This explains why we reduced our first list to six CME/shocks, and why we could not find more intervals to apply the km-TII speed analysis during these events.

The comparison of the results from our km-TII speed analysis with speeds reported by other instruments and covering different heliocentric regions verify the validity of the analysis and its potential to study the evolution of CMES/shocks with good km-TII spectral data.

This comparison of speed measurements should be done carefully. In general, we assume that the Type II radio emission is coming from the region around the shock nose, but sometimes it might come from other regions along the shock front. The near-Sun plane-of-sky LASCO-CME speeds, the shock speeds from the km-TII slope analysis, the sheet region speeds from IPS observations, and the WIND-shock and ejecta speeds from in-situ magnetic and plasma data, do not refer to the same structure but are related to the same phenomena (CME/shock event).

We have pointed out some limitations of analysing km-TII emissions to infer the shock speed. We should keep in mind that the speed values of the other instruments also present limitations. The white light plane-of-the-sky component of the CME speed detected by LASCO is not simple to deproject, by supposing a radial expansion from the source region. The reliability of the ORT and STELab IPS data would depend on the number and location of the radio sources and the conditions of the ambient solar wind where the CME/shock is propagating. IPS observations are more complicated when the medium has been perturbed by several shocks and CMEs in the preceding days. The WIND-shock and ICME localspeeds inferred from in-situ spacecraft data always present uncertainties depending on the intervals chosen to perform 
the analysis, and the local region crossing the spacecraft which do not necessarily represents the large-scale structure of the CME/shock event detected by the previous instruments.

In the analysis of the six events we find a gradual deceleration from near the Sun to $L_{1}$. We plan a comprehensive study covering more $\mathrm{CME} /$ shocks and combining white-light and remote-radio observations (combining IPS observations from different observatories) and in-situ speed measurements to illuminate the kinematics of these events.

\section{Conclusions}

The understanding of the kinematics of fast CMEs and their shocks in the interplanetary medium is a major subject for space weather purposes. We analysed WAVES/TNR spectral data associated with six CME/shocks. We applied a new technique to infer the shock propagation speed at some intervals. The results are in good agreement with previously published reports of speed values for the same events obtained by different instruments and at different heliocentric ranges. In some cases, the km-TII shock speed solutions presented significant variations. It is possible that this problem could be solved combining the analysis of simultaneous km-TII observations by different spacecraft (e.g. WIND and STEREO). If the required conditions for a good km-TII speed analysis are satisfied, the technique has good potential to be applied in future studies of CME/shock kinematics from near the Sun to $1 \mathrm{AU}$, combining the $\mathrm{km}$-TII shock speeds with other measurements.

Acknowledgements. We are grateful to M. Tokumaru of the SolarTerrestrial Environment Laboratory (STEL/STELab), University of Nagoya, Japan, for providing an updated IPS speed list of STEL observations with temporal tags. We are also grateful to Thomas McGowan Edwards for reading the manuscript. This work was partially supported by CONACyT 48494 and DGAPA PAPIIT IN110309-2 projects. The WAVES experiment on the WIND spacecraft is a joint project of the Observatoire de Paris, NASA/GSFC, and the University of Minnesota. The CME catalog is generated and maintained at the CDAW Data Center by NASA and The Catholic University of America in cooperation with the Naval Research Laboratory. SOHO is a project of international cooperation between ESA and NASA.

Topical Editor R. Forsyth thanks M. M. Bisi and another anonymous referee for their help in evaluating this paper.

\section{References}

Aguilar-Rodriguez, E., Gopalswamy, N., MacDowall, R., Yashiro, S., and Kaiser, M. L.: A universal characteristic of type II radio bursts, J. Geophys. Res., 110, A12S08, doi:10.1029/ 2005JA011171, 2005.

Bougeret, P. J., Kellogg, M. K., Manning, R., Goetz, K., Monson, S., Monge, N., Friel, L., Meetre, C., Perche, C., Sitruk, L., and
Hoang, S.: Waves: The Radio and Plasma Wave Investigation on the Wind Spacecraft, Space Sci. Rev., 71, 231-263, doi:10.1007/ BF00751331, 1995.

Brueckner, G. E., Howard, R. A., Koomen, M. J., Korendyke, C. M., Michels, D. J., Moses, J. D., Socker, D. G., Dere, K. P., Lamy, P. L., Llebaria, A., Bout, M. V., Schwenn, R., Simnett, G. M., Bedford, D. K., and Eyles, C. J.: The Large Angle Spectroscopic Coronagraph (LASCO), Solar Phys., 162, 357-402, 1995.

Cane, H. V., Sheeley, J., and Howard, R. A.: Energetic interplanetary shocks, radio emission, and coronal mass ejections, J. Geophys. Res., 92, 9869-9874, 1987.

Cremades, H., St. Cyr, O. C., and Kaiser, M. L.: A tool to improve space weather forecasts: Kilometric radio emissions from Wind/WAVES, Space Weather, 5, 8001, doi:10.1029/ 2007SW000314, 2007.

Gopalswamy, N., Lara, A., Lepping, R., Kaiser, M. L., Berdichevsky, D., and Cyr, O. C. S.: Interplanetary acceleration of coronal mass ejections, Geophys. Res. Lett., 27, 145-148, doi: 10.1029/1999GL003639, 2000.

Gopalswamy, N., Yashiro, S., Akiyama, S., Mkel, P., Xie, H., Kaiser, M. L., Howard, R. A., and Bougeret, J. L.: Coronal mass ejections, type II radio bursts, and solar energetic particle events in the SOHO era, Ann. Geophys., 26, 3033-3047, 2008, http://www.ann-geophys.net/26/3033/2008/.

Gosling, J. T., Asbridge, J. R., Bame, S. J., Hundhausen, A. J., and Strong, I. B.: Satellite Observations of Interplanetary Shock Waves, J. Geophys. Res., 73, 43, doi:10.1029/JA073i001p00043, 1968.

Manoharan, P.: Evolution of Coronal Mass Ejections in the Inner Heliosphere: A Study Using White-Light and Scintillation Images, Solar Phys., 235, 345, doi:10.1007/s11207-006-0100-y, 2006.

Manoharan, P., Tokumaru, M., Pick, M., Subramanian, P., Ipavich, F., Schenk, K., Kaiser, M., Lepping, R., and Vourlidas, A.: Coronal mass ejection of 2000 july 14 flare event: imaging from nearsun to earth enviroment, Astrophys. J. Supplement Series, 559, 1180-1189, 2001.

Manoharan, P., Gopalswamy, N., Yashiro, S., Lara, A., Michalek, G., and Howard, R.: Influence of coronal mass ejection interaction on propagation of interplanetary shocks, J. Geophys. Res., 109, 06109, doi:10.1029/2003JA010300, 2004.

Pohjolainen, S., van Driel-Gesztelyi, L., Culhane, J., Manoharan, P., and Elliott, H.: CME Propagation Characteristics from Radio Observations, Solar Phys., 244, 167-188, doi:10.1007/ s11207-007-9006-6, 2007.

Reiner, M. J., Kaiser, M. L., Fainberg, J., and Stone, R. G.: A new method for studying remote type II radio emissions from coronal mass ejection-driven shocks, J. Geophys. Res., 103, 29651, doi: 10.1029/98JA02614, 1998.

Reiner, M. J., Kaiser, M. L., and Bougeret, J.-L.: Coronal and Interplanetary Propagation of CME/Shocks from Radio, In Situ and White-Light Observations, The Astrophys. J., 663, 1369, doi: 10.1086/518683, 2007.

Richardson, I. G. and Cane, H. V.: Regions of abnormally low proton temperature in the solar wind (1965-1991) and their association with ejecta, J. Geophys. Res., 100, 23397-23412, doi: 10.1029/95JA02684, 1995.

Tokumaru, M., Kojima, M., Fujiki, K., and Yamashita, M.: Tracking heliospheric disturbances by interplanetary scintilla- 
tion, Nonlin. Processes Geophys., 13, 329-338, 2006, http://www.nonlin-processes-geophys.net/13/329/2006/.
Yashiro, S., Gopalswamy, N., Michalek, G., Cyr, O. S., Plunkett, S., Rich, N. B., and Howard, R. A.: A catalog of white light coronal mass ejections observed by the SOHO spacecraft, J. Geophys. Res., 109, A18, doi:10.1029/2003JA010282, 2004. 